\title{
How the gaze of others influences object processing
}

\author{
Cristina Becchio ${ }^{1}$, Cesare Bertone ${ }^{2}$ and Umberto Castiello ${ }^{3,4}$ \\ ${ }^{1}$ Centro di Scienza Cognitiva, Dipartimento di Psicologia, Università di Torino, Via Po 14, 10123 Torino, Italy \\ ${ }^{2}$ Centre for Theoretical and Applied Ontology, University of Turin, Via Sant'Ottavio 20, 10124 Turin, Italy \\ ${ }^{3}$ Dipartimento di Psicologia Generale, Università di Padova, Via Venezia 8, 35131 Padova, Italy \\ ${ }^{4}$ Department of Psychology, Royal Holloway-University of London, Egham, London, TW20 0EX, UK
}

\begin{abstract}
An aspect of gaze processing, which so far has been given little attention, is the influence that intentional gaze processing can have on object processing. Converging evidence from behavioural neuroscience and developmental psychology strongly suggests that objects falling under the gaze of others acquire properties that they would not display if not looked at. Specifically, observing another person gazing at an object enriches that object of motor, affective and status properties that go beyond its chemical or physical structure. A conceptual analysis of available evidence leads to the conclusion that gaze has the potency to transfer to the object the intentionality of the person looking at it.
\end{abstract}

\section{Linking the gaze of others to object processing}

From the gaze of another person we can infer information regarding both the 'outside' and the 'inside' [1-5]. With respect to the inside, we are able to access information related to intentions and mental states of another person. For instance, we can infer what another person might be interested in or what she might desire [6] and, consequently, what she might want to do next [7]. With respect to the outside, the direction of another person's gaze provides information about relevant events and objects within the environment [3]. A paradigmatic example is when the direction of an agent's gaze is followed behind a barrier. This behaviour - found in eight-month-old infants indicates an understanding that the agent is seeing something that we do not [8]. On this view, observed gaze acts as a cue to a relevant outside entity.

Previous research has considered these two aspects of gaze processing separately. Here, instead, we focus on the possible relation between them. In particular we consider the influence that intentional processing of the gaze of others might have on object processing. Our contention is that the objects falling under other people's gaze acquire properties that they would not display if not looked at.

In the first part of the article, we will examine available evidence linking others' gaze behaviour to how objects in the environment are processed. Specifically, we will discuss motor, affective and status properties that objects seem to acquire under the gaze of other people. How these properties are brought into existence will be at the core of the second part of the article. We will elaborate on the concept of 'intentional imposition' as a possible carrier of

Corresponding author: Castiello, U. (umberto.castiello@unipd.it). the influence that intentional gaze processing exerts on object processing. We shall conclude that the gaze of others has the power to impose properties that the object does not carry intrinsically, but only by virtue of an intentional imposition.

\section{Gaze processing and motor properties}

Here, we consider how others' gaze can modify the 'motor description' of an object. By 'motor description' or 'pragmatic description' we refer to a pragmatic mode of object processing - a function that allows the extraction of relevant properties from objects for action planning and control [9-11]. Evidence that the motor description of an object might be modified by the gaze of another person can be derived from kinematic studies investigating motor interference [7]. In these experiments an actor reached for a spherical target object flanked by a distractor object of a similar shape as the target, only smaller. The presence of the distractor object determined interference effects that were evident on the actor's kinematics. Once the actor completed the action, the distractor object was removed and an observer was requested to perform a similar action towards the target object presented in isolation. The logic behind these experiments was that if an automatic motor priming occurs from action observation, then interference effects should also be observed for the action subsequently performed by the observer (though the distractor was no longer present).

The results went beyond this prediction. Not only did interference transfer from the actor to the observer but also a similar effect was found in the observer's kinematics for trials in which the actor simply looked at the target flanked by the smaller distractor sphere. In other words, transfer of motor interference from another's eye direction occurred. Crucially, no transfer of interference from the human actor to the observer was revealed when the actor performed the reach-to-grasp action with the face hidden or with the eyes stationary on the target object [7]. These findings were interpreted as evidence that gaze monitoring provides information about another person's actions and motor intentions. However, an equally plausible interpretation is that the gaze of the human actor is both necessary and sufficient for activating a pragmatic representation of the distractor object. On this view, under the gaze of the actor the small sphere next to the larger sphere is not only coded as a spherical object but also becomes an object that can be 
grasped. In the terms of object processing, the object is enriched by a motor component imposed by other people's gaze (see Box 1).

\section{Gaze processing and emotional properties}

Recently, a new line of research concerned with the impact that the gaze of others might have on the evaluative judgements of objects has emerged [12,13]. The main question is: do we prefer objects that are looked at by other people? To address this issue participants were presented with a face looking towards or away from an object [12]. It was found that objects that are looked at are more likeable than those that do not receive much attention from others. This suggests that the gaze behaviour of others might have an impact on our affective appraisal of objects in the environment.

To investigate whether this liking effect was modulated by the emotional expression of the observed face, a subsequent study [13] compared affective ratings of objects that were looked at with a happy or a disgusted face. The results spoke clearly. Objects that were viewed with a happy expression were rated as more likable than were objects that were looked at by a face expressing disgust. This occurred despite attention being similarly cued in the direction of the observed gaze by happy and disgusted faces.

Finally, the affective power of observed gaze was confirmed by investigating the influence that the quality of a motor interaction might have on object affective ratings [14]. Participants observed an actor grasping and moving objects by using either a fluent or a non-fluent action. In one condition the observed actor could be seen while she was looking towards the to-be-grasped object, whereas in another condition the actor's head and gaze could not be seen. In line with previous studies [12,13], when the actor's gaze could not be seen, liking ratings of objects were reduced. Further, liking ratings were higher for fluent actions but only when the actor's gaze towards the object

\section{Box 1. When gaze turns into grasp: neural evidence}

Evidence that motor intentions can be inferred from gaze direction has been provided by using event-related functional magnetic resonance imaging (fMRI). In one study [31] participants observed videos showing a human model either grasping a target object (grasping condition) or simply gazing (gaze condition) at the same object. These two conditions were contrasted with each other and also against a control condition in which the human model was standing behind the object without performing any gazing or grasping action. The results revealed activation within the dorsal premotor cortex, the inferior frontal gyrus, the inferior parietal lobule, and the superior temporal sulcus in both 'grasping' and 'gaze' conditions (Figure $(a, b)$, suggesting that a common intentional system might underlie the representation of both hand-object relations and gaze-object relations. This conclusion was confirmed in a second study [32] in which participants observed a human model gazing towards a target object presented either in isolation or flanked by a distractor object. First, results confirmed that the observation of gaze shifts directed towards a target object yielded activation within areas of the brain that are commonly known to be involved in coding hand-object interactions. Second, when gaze occurred in the presence of a distractor object a decrease of activation was revealed within the inferior frontal gyrus. This decrease of signal change closely resembles the signal change modulation previously reported within the inferior frontal gyrus during the observation of interfered hand actions [33].
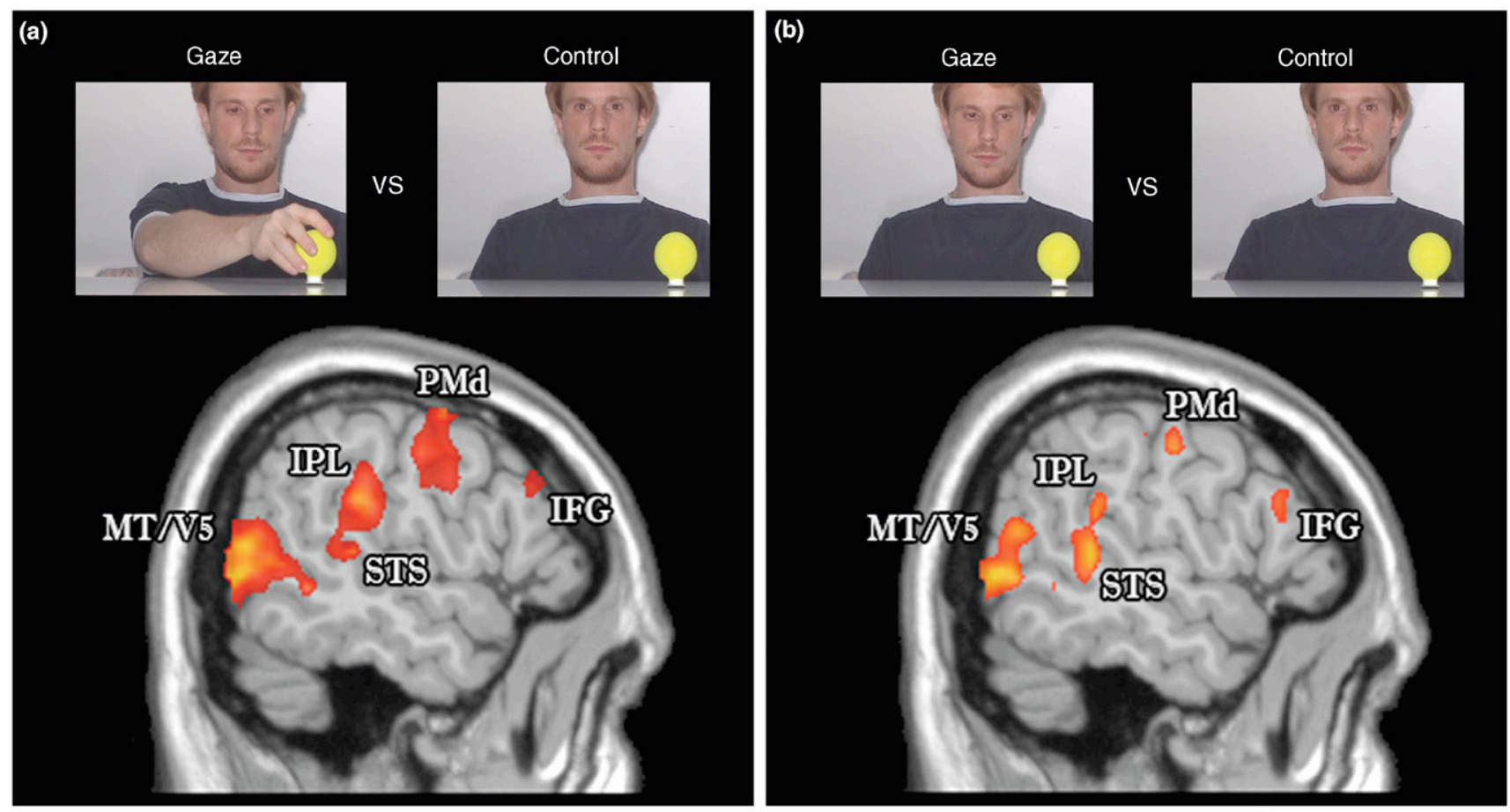

TRENDS in Cognitive Sciences

Figure I. Neural activity related to the comparison between the grasping versus the control condition (a) and the gaze versus the control condition (b). Modified, with permission, from reference [21]. 
was visible. Altogether, these findings provide evidence that the gaze of others can invest objects with affective properties.

\section{Gaze processing and status properties}

The degree of novelty assigned to an object is another aspect that could be influenced by observed gaze. Intuitively, an object that has never been seen before appears less familiar than an already known object. But what about an object looked at by another person? Will it appear more familiar than an object that has not attracted the attention of others? These questions have been investigated by using a behavioural novelty preference paradigm with infants [15]. In these experiments infants watched a video presentation of an adult gazing towards one of two objects. Then a paired-preference test phase began. During this phase the same objects were presented but in the absence of the adult gazing at them. It was found that infants looked reliably less at the previously 'gazed' object, suggesting that the object cued by the adult's gaze was perceived as less novel than the non-cued object. Similar results have been obtained in an event-related potential (ERP) study by using a similar paradigm [16]. It was found that an ERP wave, previously found to be sensitive to differences in processing of familiar and unfamiliar objects in infants [17,18], was larger for the non-cued than for the cued test trials. Thus, the cued object was processed as more familiar than the non-cued object. These data indicate that the infants' processing of objects is influenced by others' gazes. More specifically, the data demonstrate that the status of objects - familiar versus unfamiliar - is modified by the looking behaviour of others.

\section{How gaze processing affects object processing}

Building on the concept of secondary intersubjectivity developed by Trevarthen [19] it has been posited that it is not just people that are considered special but also the objects with which other people interact [20]. The aforementioned evidence suggests that this is especially true for those objects other people look at. Under the gaze of others, objects acquire properties that they would not display if not looked at, namely motor, affective and status properties. How do these properties arise? What is the mechanism responsible for this 'enriching' effect? Below we consider three issues that, in our opinion, need to be considered for providing possible answers to these questions.

\section{Beyond an attentional shift}

The first issue is whether this effect might simply reflect a cueing effect. Observing another person looking at an object automatically shifts our attention to the viewed object [21]. This raises the question of whether the assignment of novel properties to an object might occur because the agent draws more attention to it. We contend that such explanation is unlikely because although similar cueing effects are evoked by arrow cues, they do not seem to influence object processing [12]. As gaze cues, symbolic cues such as arrows are effective in automatically triggering attentional orienting [22]. However, unlike gaze cues, they do not guarantee a consequence on object processing. For instance, no modulation was observed in the affective response to objects when arrow cues were used rather than gaze cues [12]. Because gaze and arrow cues produced a similar cueing magnitude, this implies that attentional cueing is not, per se, responsible for the effect on object processing.

\section{Intentional gaze processing}

The second issue is concerned with the intentionality of perceived gaze. The enriching effect does not appear to be based on gaze processing, per se, but seems to result from the intentionality of perceived gaze, i.e. observing another person looking at an object. As reported above, this variable has been manipulated by asking a model to fixate on the target and not to look at a distractor stimulus [7]. In this condition no effect of perceived gaze was revealed on object processing. Similarly, no affective effects on object processing have been revealed when another person gazes away from an object [12]. Taken together, these findings suggest that the enriching effect is specifically related to intentional gaze processing, with intentional gaze being a necessary condition for the effect to arise.

\section{Persistence}

The third issue is whether properties such as attractivity should be treated as properties of objects or properties of others' perceived gaze. One reason to ascribe affective, status and motor properties to objects, rather than to others' gaze, is that these properties seem to persist even when the gaze of others is no longer visible. In other words when under the gaze of others, objects become graspable, attractive or familiar. Once these properties have been attached to the object, they no longer require the observer being present to be effective.

\section{Intentional imposition: transferring intentionality onto objects}

The three issues considered earlier suggest the existence of a mechanism that allows transferring to an object the intentionality of the person who is looking at it. A mechanism through which this transfer could take place is 'intentional imposition'.

When theorizing about intentionality, philosophers distinguish between original or intrinsic intentionality and derived or extrinsic intentionality. Although only mental states exhibit original intentionality, objects might carry a derived intentionality (i.e. a kind of intentionality that is imposed onto objects by intentional agents [23]). On this view, intentional imposition enriches the object with properties that the object itself did not previously show. These properties are observer dependent in the sense that they depend, for their very existence, on intentional agents [24] (see Box 2).

Although in current philosophical debate the notion of intentional imposition applies mainly to social and institutional objects $[24,25]$, the evidence reported earlier seems to suggest that at a more basic, noninstitutional level, a similar mechanism of intentional imposition could operate through observed gaze. When considering the context of social interactions, any object falling under the gaze of others can acquire novel observer-dependent properties (i.e. properties that the object would not display if not looked at by an intentional agent). 


\section{Box 2. Observer-dependent features}

Observer-dependent features depend on observers for their very existence, thus differing from observer-independent features, such as mass or force, which exist independently of observers. More specifically, observer-dependent features are created by observers in the discharge of their intentionality $[24,25]$. In dealing with observer-dependent features, an important distinction is between what we might call subjective dependent features and social and institutional features [24]. Both subjective dependent features and social and institutional features depend on the intentionality of observers and are, thus, observer relative. The intentionality they depend on is different, however: whereas subjective dependent features depend on individual intentionality, social and institutional features depend on collective intentionality [24]. Examples of subjective dependent features are the sort of features that objects derive from being looked at, such as being familiar or attractive. These properties depend on the specific attitude read in the other's individual gaze. Examples of collective dependent features are it 'counting as a twenty dollar bill' or performing the function of a boundary marker. These features do not depend on the intentionality of any individual but on the fact that we, collectively, assign a certain status to an object (e.g. a piece of paper or a yellow line) that enables the object to perform a function that could not be performed without the collective acceptance of the status $[24,25]$.

A first prediction stemming from such interpretation is that the property transferred to an object should depend on the specific attitude or intention inferred from the gaze of others. Although no experimental work has specifically tested such a hypothesis, the findings by Bayliss et al. [12] seem to confirm this prediction by showing that objects attended by others are evaluated according to the positive or negative valence of the emotions transmitted by gaze. Further evidence can be drawn from the studies in which gaze is used as an indicator of the model's future action [7]. In line with the 'intentional imposition' hypothesis, the property that the object acquires under this condition is that of being graspable.

Following the same logic, it might be predicted that when intentional gaze processing is absent or deficient, this should not only prevent the representation of the mental states of others but also impoverish the object representation. This is exactly what seems to be happening in individuals with autism [26]. Individuals with autism fail to use gaze to infer another person's intentions [27-30]. By using a motor interference paradigm, it has been demonstrated that such inability impacts on how individuals with autism process objects looked at by other people. Whereas the actions of normally developing children are affected by the presence of a distractor object looked at by an observer, children with autism do not show any interference effect. This suggests that whereas normally developing children inevitably form a motor representation of objects falling under the gaze of an observer, in individuals with autism object processing is immune to any influence of observed gaze [26]. A point worth noting is that autistic children do not exhibit a generalized object-processing deficit, but the deficit is specifically confined to the processing of motor features that are imposed on objects by the observer's intentionality.

\section{Concluding remarks}

What is an object? What counts as an object? If we consider our daily experience, the properties displayed by objects go

\section{Box 3. Outstanding questions}

- The influence of gaze processing on object processing has not been systematically investigated. Are there other properties outside motor, affective and status properties that observing the gaze of others on objects might trigger? What are the most appropriate techniques and paradigms to explore such a relationship more thoroughly?

- In neural terms we already have some evidence of which neural substrates are involved in the coding of object motor properties triggered by gaze. The natural question is, what are the neural underpinnings of derived affective and status properties of objects?

- What is the relationship between the derived and intrinsic properties of an object? Does the brain treat derived object properties in a similar fashion to intrinsic object properties?

- The persistence of derived object properties is an important question. We know that they survive even when the gaze of others is no longer available, but how long do they persist?

- Are the processes described here special to gaze or do they also arise for other biological cues?

- Preliminary evidence suggests that observed gaze does not trigger motor properties in individuals with autism. Does this also apply to affective and status properties?

- What is the role this process might play for the construction of a social reality?

beyond their physical and chemical structure. Objects can appear as familiar or unfamiliar, can have a value or can be associated with a possibility of action. To account for these properties, we need to consider humans' capacity to transfer observer-dependent properties to objects in which the object does not carry the property intrinsically but only by virtue of the intentional imposition. The idea outlined here is that at a basic level an important source of observerdependent properties might be found in other people's gaze. The gaze of others doesn't only have the effect of shifting our attention towards the observed object: under the gaze of others the object is enriched with motor, emotive and status components that it would not display if not looked at. This raises several interesting questions to be pursued in future research spanning from the revelation of the neural mechanisms underlying such processes to the implications such processes might have for specific subdisciplines, such as the study of autism (see Box 3).

\section{Acknowledgements}

This work was supported by a grant to U.C. from the Italian Ministry of the University and Research. C.B. was supported by the Fondazione Cassa di Risparmio di Torino.

\section{References}

1 Allison, T. et al. (2000) Social perception from visual cues: Role of the STS region. Trends Cogn. Sci. 4, 267-278

2 Frith, C.D. and Frith, U. (2007) Social cognition in humans. Curr. Biol. 17, R724-R732

3 Frischen, A. et al. (2007) Gaze cueing of attention: visual attention, social cognition, and individual differences. Psychol. Bull. 133, 694-724

4 Langton, S.R.H. et al. (2000) Do the eyes have it? Cues to the direction of social attention. Trends Cogn. Sci. 4, 50-59

5 Pelphrey, H.A. and Morris, J.P. (2005) Brain mechanisms for interpreting the actions of others from biological-motion cues. Curr. Dir. Psychol. Sci. 15, 136-140

6 Lee, K. et al. (1998) Children's use of triadic eye gaze information for "mind reading". Dev. Psychol. 34, 525-539

7 Castiello, U. (2003) Understanding other people's actions: intention and attention. J. Exp. Psychol. Hum. Percept. Perform. 29, 416-430 
8 Csibra, G. and Volein, A. (2008) Infants can infer the presence of hidden objects from referential gaze information. Brit. J. Dev. Psychol. 26, 1-11

9 Jeannerod, M. et al. (1995) Grasping objects: the cortical mechanisms of visuomotor transformation. Trends Neurosci. 18, 314-320

10 Milner, A.D. and Goodale, M.A. (1995) The Visual Brain in Action. Oxford University Press

11 Milner, A.D. and Goodale, M.A. (2008) Two visual systems re-viewed. Neuropsychologia 46, 774-785

12 Bayliss, A.P. et al. (2006) Gaze cueing and affective judgments of objects: I like what you look at. Psychon. Bull. Rev. 13, 1061-1066

13 Bayliss, A.P. et al. (2007) Affective evaluations of objects are influenced by observed gaze direction and emotional expression. Cognition 104, 644-653

14 Hayes, A.E. et al. (2007) Self produced and observed actions influence emotion: the roles of action fluency and eye gaze. Psychol. Res. 72, 461472

15 Reid, V.M. and Striano, T. (2005) Adult gaze influences infant attention and object processing: implications for cognitive neuroscience. Eur. J. Neurosci. 21, 1763-1766

16 Reid, V.M. et al. (2004) Eye gaze cueing facilitates neural processing of objects in 4-month-old infants. Neuroreport 15, 2553-2555

17 de Haan, M. and Nelson, C. (1999) Brain activity differentiates faces and object processing in 6-month-old infants. Psychol. Sci. 9, 131-134

18 Nelson, C.A. (1997) Electrophysiological correlates of memory development in the first year of life. In Biological and Neuropsychological Mechanisms (Reese, H.W. and Franzen, M.D., eds), pp. 95-131, Erlbaum
19 Trevarthen, C. (1979) Communication and cooperation in early infancy: a description of primary intersubjectivity. In Before Speech (Bullowa, M., ed.), pp. 321-372, Cambridge University Press

20 Hobson, P. (2002) The Cradle of Thought. Macmillan

21 Friesen, C.K. et al. (2005) Does gaze direction really trigger a reflexive shift of spatial attention? Brain Cogn. 57, 66-69

22 Tipples, J. (2002) Eye gaze is not unique: Automatic orienting in response to uninformative arrows. Psychon. Bull. Rev. 9, 314-318

23 Searle, J. (1983) Intentionality. An Essay in the Philosophy of Mind. Cambridge University Press

24 Searle, J. (1995) The Construction of Social Reality. Free Press

25 Searle, J. (2006) Social ontology: some basic principles. Anthropol. Theor. 6, 12-29

26 Becchio, C. et al. (2007) Motor contagion from eye gaze. The case of autism. Brain 130, 2401-2411

27 Baron-Cohen, S. (1995) Mindblindness: an Essay on Autism and Theory of Mind. MIT Press

28 Dawson, G. et al. (1998) Neuropsychological correlates of early symptoms of autism. Child Dev. 69, 1277-1285

29 Frith, C.D. and Frith, U. (1999) Interacting minds-a biological basis. Science 286, 1692-1695

30 Leekam, S.R. et al. (1998) Targets and cues: gaze-following in children with autism. J. Child Psychol. Psyc. 39, 951-962

31 Pierno, A.C. et al. (2006) When gaze turns into grasp. J. Cogn. Neurosci. 18, 2130-2137

32 Pierno, A.C. et al. (2008) Motor ontology in representing gaze-object relations. Neurosci. Lett. 430, 246-251

33 Pierno, A.C. et al. (2006) Transfer of interfered motor patterns to self from others. Eur. J. Neurosci. 23, 1949-1955 